\title{
Professionalisierung DES TRINATIONALEN BEgEgNUNGSPROJEKTES IM DREILÄNDERECK D-PL-CZ ANHAND DER WISSENSCHAFTLICHEN BEGLEITUNG
}

\author{
Sînziana Schönfelder ${ }^{1}$; Emilia Socha ${ }^{2}$ \\ Technische Universität Dresden, IHI Zittau, \\ Professur für Produktionswirtschaft und Informationstechnik, \\ Markt 23, 02763 Zittau, Deutschland \\ e-mail: ${ }^{1}$ alina_sinziana.schoenfelder@tu-dresden.de; ${ }^{2}$ emilia.socha@tu-dresden.de
}

\begin{abstract}
Das Begegnungsprojekt „Art and Science - Innovative Lernformen in der grenzüberschreitenden Zusammenarbeit im Dreiländereck D-CZ-PL“ stellt eine Kooperation zwischen dem Internationalen Hochschulinstitut (IHI) Zittau - TU Dresden, der Hillerschen Villa e.V. in Zittau und dem Science Center IQ Landia in Liberec (CZ) dar. Das Ziel der wissenschaftlichen Begleitung besteht in der Qualitätssicherung mittels einer Evaluation von inter- und transkulturellen Kompetenzen bei den teilnehmenden Jugendlichen sowie einer qualitativen Erhebung hinsichtlich der Fortbildungsbedarfe bei den beteiligten Multiplikatoren. Der Beitrag beschreibt die ersten Zwischenergebnisse aus den qualitativen und quantitativen Befragungen der wissenschaftlichen Begleitung sowie daraus resultierende Handlungsempfehlungen.
\end{abstract}

\section{Keywords}

Inter- and Transcultural competence; Border Triangle; Transnational projects; Children and youth work; Cross-border cooperation; Non-formal learning, Professionalisation of encounter work.

\section{Einleitung}

Im vorliegenden Beitrag werden die ersten Zwischenergebnisse der wissenschaftlichen Begleitung im Rahmen des Projektes „Art and Science - Innovative Lernformen in der grenzüberschreitenden Zusammenarbeit im Dreiländereck D-CZ-PL“" vorgestellt. Die Laufzeit des Projektes Art and Science beträgt drei Jahre. Von Oktober 2017 bis September 2020 sollen insgesamt bis zu 950 Jugendliche daran teilnehmen. Neben dem IHI Zittau - TU Dresden, sind am Projekt die Hillersche Villa e.V. als Lead Partner und das Science Center IQ Landia (CZ) als Projektpartner beteiligt. Das Projekt wird aus Mitteln der Europäischen Union im Rahmen des Europäischen Fonds für Regionale Entwicklung realisiert. Dieses Pilotprojekt soll in der Grenzregion als Modell für inter- und transkulturelle Bildung und Begegnung etabliert werden.

\section{Forschungsgegenstand}

Im grenzübergreifenden Projekt finden im Rahmen von künstlerischen und naturwissenschaftlichen Werkstätten Begegnungen zwischen Jugendlichen im Dreiländereck D-CZ-PL statt. Dabei sollen die teilnehmenden Jugendlichen inter- und transkulturelle Kompetenzen erwerben. Um die Qualität der Begegnungsformate zu gewährleisten, werden bedarfsorientierte Fortbildungen für Werkstattleiter/innen, Sprachmittler/innen und Lehrer/innen angeboten. 
In einer Werkstattwoche arbeiten parallel bis zu 15 Schüler aus jeweils drei Schulen, aus Deutschland, Tschechien und Polen in bis zu sieben medialen Lernformaten zusammen: Musik, Theater/Tanz, Fotografie, Text, Film und Aktionskunst. [Vgl. 8] Während der Werkstatt setzen sich die Teilnehmer/Innen mit einem vorgegebenen Thema auseinander, um am Ende ein gemeinsames Kunstprodukt zu entwickeln. Dabei erlernen sie das notwendige künstlerische Handwerk und erlangen inter- und transkulturelle Kompetenzen. Bei der Gruppenarbeit spielen die Sprachmittler/Innen (polnisch, tschechisch und deutsch) eine Schlüsselrolle. Am Ende der Werkstattwoche wird die jeweilige Kunstproduktion (z. B. Fotoausstellung, Theaterstück, Kurzfilm, Performance, etc.) öffentlich an zwei bis drei Orten gezeigt.

Die bei Art and Science vorhandenen Lernformate basieren auf dem Erfahrungshintergrund des Projektes Lanterna futuri, das seit 2004 Bildungs- und Begegnungsarbeit im Dreiländereck D-PL-CZ betreibt. Der Versuch, Kunst und Technologie zu verbinden und auf Lernformate zu übersetzen, bildet den innovativen Charakter von Art and Science.

Die Rolle der wissenschaftlichen Begleitung des IHI Zittau - TU Dresden besteht zunächst darin, die Aneignung von inter- und transkulturellen Kompetenzen zu evaluieren und die Fortbildungsbedarfe bei Werkstattleiter/innen, Sprachmittler/innen und Lehrer/innen zu ermitteln. Des Weiteren leitet die wissenschaftliche Begleitung Handlungsempfehlungen aus den zusammengeführten Ergebnissen ab, wobei die Umsetzung der in der Handlungsverantwortung des Leadpartners liegt.

\section{Theoretischer Orientierungsrahmen}

Nach der Analyse der bisherigen Projektpraxis sowie der dahinterliegenden theoretischen Ansätze wurde die Grundlage für die Forschungsarbeit gelegt. Dabei mussten im Vorfeld zahlreiche Definitionen von elementaren Begriffen, wie z. B. der, ,interkulturellen Kompetenz", vorgenommen werden. Hierbei war es notwendig, dass die Komponenten, aus denen sich interkulturelle Kompetenz im Projekt Art and Science zusammensetzt, erneut bestimmt werden. Ebenso sollten die Lernziele einer Art and Science Werkstattwoche festgelegt werden.

Im Projekt Art and Science wird mit dem Begriff der Interkulturalität operiert und demnach den interkulturellen Kompetenzen, welche Moosmüller als ,die allgemeine linguistische, soziale und psychische Fähigkeit einer Person, mit Individuen und Gruppen, die einer anderen Kultur angehören, erfolgreich zu kommunizieren" [3:272] bezeichnet. In dem theoretischen Orientierungsrahmen des Projekts fließen allerdings Aspekte der Transkulturalität mit ein, indem das Kulturübergreifende in den Vordergrund rückt. Auf beide Begriffe wird im Projekt zurückgegriffen, wobei hauptsächlich die Bezeichnung „,interkulturell“ verwendet wird. Um die unterschiedlichen Bedeutungen der Begriffe zu verstehen, die den beiden Termini zugeschrieben werden, ist eine Auseinandersetzung mit dem Kulturbegriff unerlässlich.

Am Begriff ,interkulturell“ wird kritisiert, dass er sich auf das obsolete primordiale Kulturverständnis gründet. Das Präfix ,inter" würde darauf hinweisen, dass von mindestens zwei klar abgrenzbaren Kulturen im Herderschen Sinn ausgegangen werde, und dadurch das Trennende, und Unterschiedliche zwischen den Kulturen betont würde. [vgl. 7:46f.] Welsch hat diesem Kulturkonzept das Konzept der Transkulturalität entgegengestellt, ,das deskriptiv und normativ ein anderes Bild vom Zustand und Verhältnis der Kulturen entwirft: eines nicht der Isolierung und des Konflikts, sondern der Verflechtung, Durchmischung und Gemeinsamkeit." [5:10] Das Konzept basiert auf einer dynamischen Auffassung des 
Kulturbegriffs. „Kulturen” werden demnach nicht als homogene Einheiten verstanden, sondern als offene Systeme, die unter wechselseitigen Einflüssen stehen.

Da das Projekt Art and Science sowohl vom Konzept der „Interkulturalität“ als auch „Transkulturalität“" gespeist wird, wird im Folgenden von beiden Begriffen Gebrauch gemacht.

\section{$3 \quad$ Forschungsmethoden}

Die Evaluation des Kompetenzerwerbs bei den Jugendlichen und die Erhebung der Fortbildungsbedarfe erfolgen in zwei Untersuchungsperioden. Das Forschungsdesign der wissenschaftlichen Begleitung basiert auf einer Triangulation qualitativer und quantitativer Verfahren, die mittels Fragebögen, problemzentrierten Interviews, Teilnehmender Beobachtung sowie Gruppendiskussionen umgesetzt wird.

\subsection{Qualitative Erhebung}

Zum einen soll die qualitative Erhebung die Fortbildungsbedarfe der Werkstattleiter/innen sowie der Sprachmittler/innen und der Lehrer/innen ermittelt werden. Zum anderen soll die qualitative Erhebung die notwendigen Entwicklungsschritte des Projektes aus Sicht der handelnden Akteure/innen ergründen.

Für die qualitative Erhebung wurde die Methode des problemzentrierten Interviews ausgewählt. Alle zehn durchgeführten Interviews wurden aufgenommen, transkribiert und anonymisiert. Bei der Auswahl der Interviewpartner/innen wurde darauf geachtet, die vorhandene Heterogenität der vorhandenen Akteure/innen abzubilden. Es wurden Werkstattleiter/innen, Sprachmittler/innen und Lehrer/innen interviewt. Die ausgesuchten Interviewpartner/innen sollten alle drei Sprachregionen (deutsch, tschechisch, polnisch) vertreten und über eine unterschiedlich lange Mitwirkung im Projekt Lanterna futuri verfügen. Da die Gruppe der Lehrer/innen noch unzureichend untersucht wurde, wird auf diese im vorliegenden Beitrag nicht näher eingegangen.

Anhand eines Leitfadens wurden folgende Themen(Auswahl) angesprochen:

- Beschreibung des Projektes und der eigenen Rolle

- Allgemeine sowie spezifische Weiterbildungsbedarfe

- Motivation im Projekt zu arbeiten

- Fähigkeiten einer/eines Werkstattleiter/in bzw. einer/eines Sprachmittler/in

- Fragen zur Methodik (erfahrungsorientiert vs. methodisch orientiert)

- Herausforderungen und Verbesserungspotentiale

- Weiterempfehlung des Projektes

Die transkribierten Leitfadeninterviews wurden mit Hilfe der Software MaxQDA ausgewertet. Die Codierung der Interview-Daten erfolgte durch Zuordnung einzelner Textabschnitte zu Kategorien. Hierbei wurde keine rein induktive, also aus den Daten abgeleitete, oder deduktive, d.h. im Voraus theoriegeleitet entwickelte, Kategorienbildung [Vgl. zu dieser Unterscheidung z. B. 2:74f.] vorgenommen, sondern eine Mischform bevorzugt. Bei dieser werden ,aus der Fragestellung und theoretischen Traditionen [...] abgeleitete, zunächst eher vage Kategorien" [4:548] im Verlauf der Analyse in der Auseinandersetzung mit dem Untersuchungsmaterial ausdifferenziert, mit neuen Kategorien ergänzt oder auch ersetzt. 


\subsection{Quantitative Erhebung}

Im Rahmen der quantitativen Befragung wird untersucht, inwiefern Teilnehmer/innen einer Art and Science Werkstattwoche inter- und transkulturelle Kompetenzen, bezogen auf folgende sechs Dimensionen, erlangen:

- soziale Kompetenzen im interkulturellen Kontext (Kommunikationsfähigkeit, Teamfähigkeit, Konfliktfähigkeit, Kritikfähigkeit)

- Einüben des Perspektivenwechsels

- Aneignung und Beschaffung von Wissen

- Fördern von Einstellungen und Werten (Toleranz, Offenheit, Akzeptanz)

- Reflektieren von Situationen des interkulturellen Umgangs

- Erkennen und Überwinden von Ethnozentrismus

Die oben genannten Dimensionen entstanden in Anlehnung an einer Studie zur Vermittlung interkultureller Kompetenz, welche u.a. von Ursula Bertels im Jahr 2004 durchgeführt wurde [vgl. 1:37].

Die Untersuchung setzt sich aus vier aufeinanderfolgenden und sich zum Teil überschneiden Arbeitsphasen zusammen: Vorbereitungsphase, Durchführungsphase, Nachbesserungsphase, Evaluationsphase. Während der Vorbereitungsphase wurden die konkreten Methoden zur Datenerhebung konzipiert. Die Vorgehensweise der quantitativen Befragung wurde während der Durchführungsphase im März 2018 erprobt und bei der Nachbesserungsphase bis August 2018 angepasst. Die abschließende Evaluation steht noch bevor.

\section{$4 \quad$ Darstellung der ersten Zwischenergebnisse}

Aufgrund der nicht abgeschlossenen Untersuchungsperiode sowie der begrenzten Seitenzahl erfolgt eine auszugsweise Darstellung der ersten Zwischenergebnisse. Hier sollte ergänzt werden, dass die Untersuchungsperiode I noch nicht abgeschlossen ist, d.h. dass die Durchführung der Interviews sowie der quantitativen Befragung zum aktuellen Zeitpunkt fortschreitend erfolgt.

\subsection{Qualitative Erhebung}

Im Folgenden werden die zentralen Komponenten aus der Auswertung der transkribierten Interviews erläutert:

\subsubsection{Uneinheitliches Projektverständnis}

Im Rahmen des Projektes werden die an Jugendlichen zu vermittelnden Kompetenzen und Inhalte differierend beschrieben. Die Notwendigkeit einer gemeinsamen konzeptionellen Ebene wird anhand des Zitats dargelegt:

„(...) Was ist // was ist unsere Philosophie? Wie sehen wir die Welt [ERNEUT RUFE IM HINTERGRUND] und was wollen wir tatsächlich an Inhalten vermitteln? Also ich find die Methoden da eher zweitrangig, ich find da im // in erster Linie wichtig uns nochmal über die Inhalte zu verständigen. Das machen wir ein Stück weit _" [9]

Dabei geht es um Inhalte, die über das Thema der Werkstätte hinaus eine Bedeutung haben.

„....Eine Lanterna Philosophie. Weil die Diskussion kam jetzt auch bei den Werkstattleitern öfter mal so ins [VÖGELKREISCHEN ODER KRÄHEN IM HINTERGRUND] // ins Rollen. So am Rande zwar, aber es war spürbar, dass irgendwie // dass wir uns da nochmal ºisschen verständigen müssen. “ [9] 
Das uneinheitliche Verständnis bezieht sich sowohl auf die Ebene der Inhaltsvermittlung als auch auf die Ebene einer grundlegenden Projektauffassung, hinsichtlich Beschreibung, Ziele und Inhalte von Art and Science (früher Lanterna futuri).

\subsubsection{Wissenstransfer Werkstattleiter/innen erwünscht}

Im Projekt wirken Werkstattleiter/innen mit einer Vielzahl an Qualifikationen und Erfahrungen in der interkulturellen Arbeit. Es wird kritisiert, dass die Möglichkeiten zum Austausch untereinander begrenzt sind. So heißt es in einem Interview:

„(...) wir haben wenig Momente, wo wir Zeit haben uns in Ruhe auszutauschen. So, als Werkstattleiter. Wir haben gestern mal kurz eine Viertelstunde zusammen einfach rumgesessen. Und das war das einzige Mal, dass wir // sonst treffen wir uns beim Mittagessen. Wir haben alle so viel zu tun, [*] dass es ei/l [*] dass es [*] wenig Momente gibt, wo wir mal ohne eine Team-Sitzung, sondern so ganz entspannt ins Gespräch kommen und man sich darüber mal austauschen kann. Finde ich sehr schade, weil ich glaube, wie Du sagst, es wäre produktiv. Weil [**] jetzt // ich kenn ja jetzt nur X und $J$ als andere Werkstattleiter und ich finde das sehr interessant, wie die arbeiten. Und [*] seh die aber // also [**] wir können nur manchmal sagen ,hey, bei uns ist gerade das los. Und bei uns ist gerade das Problem. “ Dann sagen wir „Ja, ich wüsste noch das Spiel, vielleicht kannst Du das mal ausprobieren." So etwas findet schon statt, aber so, dass man sich mal ganz in Ruhe über Methoden unterhalten kann, das haben wir bisher nicht gehabt" [11]

In einem anderen Interview wird bekräftigt, dass ein grundsätzliches Interesse besteht, von der Arbeit anderer Werkstattleiter/innen zu lernen:

„Oder, dass die // die Workshopleiter Vorträge über ihre Arbeit halten. Das find ich auch wichtig. Ich weiß gar nicht wie der Werkstattleiter X seine Typen motiviert, dass sie ein Gedicht schreiben. Interessiert mich aber. " [12]

Die Vielfalt der Austauschbereiche wird thematisiert, allerdings werden die fehlenden Möglichkeiten dazu bemängelt:

„... da sehe ich auf jeden Fall Verbesserungspotenzial auch. [*] Ich finde schon, dass wir eine Teamkultur haben, [*] aber mehr Austausch wäre super [*]. Vor allem zum Thema [*] eben Methodik/Umgang mit dem Thema; Einstieg ins Thema; usw. und so fort. Also, es // die Bandbreite ist riesig wo man sich austauschen kann" [11]

\subsection{Quantitative Erhebung}

Die Untersuchung der Entwicklung der Kompetenzen der Jugendlichen läuft mehrstufig ab. Die Jugendlichen werden vor und nach einer Werkstattwoche anhand von dreisprachigen Fragebögen befragt. An der Probebefragung im März 2018 nahmen alle 63 Teilnehmer/Innen im Alter von 13 bis 18 Jahren teil. Die Verteilung der Nationalitäten ist wie folgt ausgefallen: 21 aus Deutschland, 23 aus Tschechien,16 aus Polen und 2 aus der Ukraine. Teil. Die zuletzt Genannten gehörten zur polnisch-sprachigen Gruppe dazu. Die Daten wurden vor Ort an den Begegnungsorten Liberec $(39,68 \%$ der Befragten) und Großhennersdorf $(60,32 \%$ der Befragten) direkt am ersten und am letzten Tag der Werkstattwoche erhoben. Die befragte Zielgruppe nahm an folgenden Werkstätten teil: Aktionskunst, Musik, Theater, Film und Fotografie. Erwähnenswert ist, dass 38 der Befragten bisher an keinen anderen internationalen Begegnungsprojekten teilgenommen haben. 
Für diesen Abriss wurde die Auswertung von drei Items aus den bei der Werkstattwoche im März ausgefüllten Fragebögen ausgewählt.

\subsubsection{Fremdsprachenkenntnisse}

Bei der Frage: „Wie schätzt du deine Polnisch-Sprachkenntnisse ein?“ reichte die Skala von „Keine Sprachkenntnisse“ bis „Muttersprache“. Dabei kreuzten 38 der insgesamt 62 Befragten an, dass sie über keine Polnisch-Sprachkenntnisse verfügen und 6 schätzten sich als „Anfänger" ein. 12 kreuzten an, dass sie Muttersprachler sind, 4 ,sehr gut", 2 , ,gut" und eine Person machte keine Angabe. (Fig. 1 - Abb. 1)

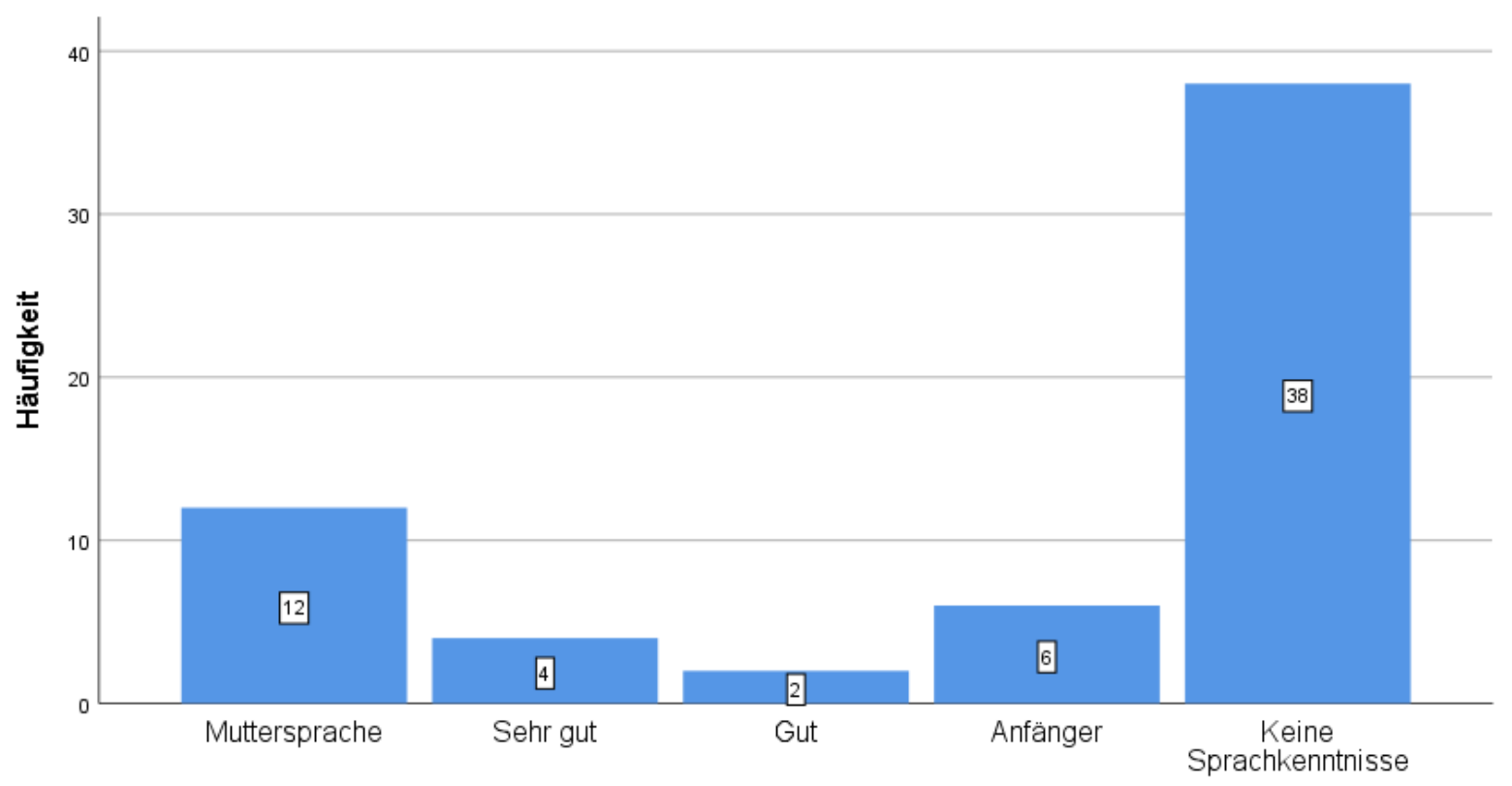

Quelle: Eigene Darstellung

Fig. 1: Vor der Werkstattwoche: „Wie schätzt du deine Polnisch-Sprachkenntnisse ein?“ Absolute Werte!

Im Vergleich dazu wurde am letzten Tag der Werkstattwoche erneut die Frage nach den Polnisch-Sprachkenntnissen gestellt. Diesmal waren es nur 12 von den insgesamt 59 Befragten, die „Keine Sprachkenntnisse“ angekreuzt haben. Weitere 23 Befragte schätzten sich selbst als „Anfänger" ein. Andere 3 Befragte stuften ihre Polnisch-Kenntnisse als „Gut“, 3 als „Mittel" und 2 als „Fundierte Grundkenntnisse“ ein. Das Ergebnis deutet darauf hin, dass die Teilnehmer der Werkstattwoche in ihrer subjektiven Wahrnehmung Kompetenzen in Fremdsprachen erlangen und wie hier am Beispiel Polnisch sichtbar geworden ist, 26 Befragte von ,keinen Sprachkenntnissen“ zu „Anfängern“ übergegangen sind. (Fig. 2 - Abb. 2) 


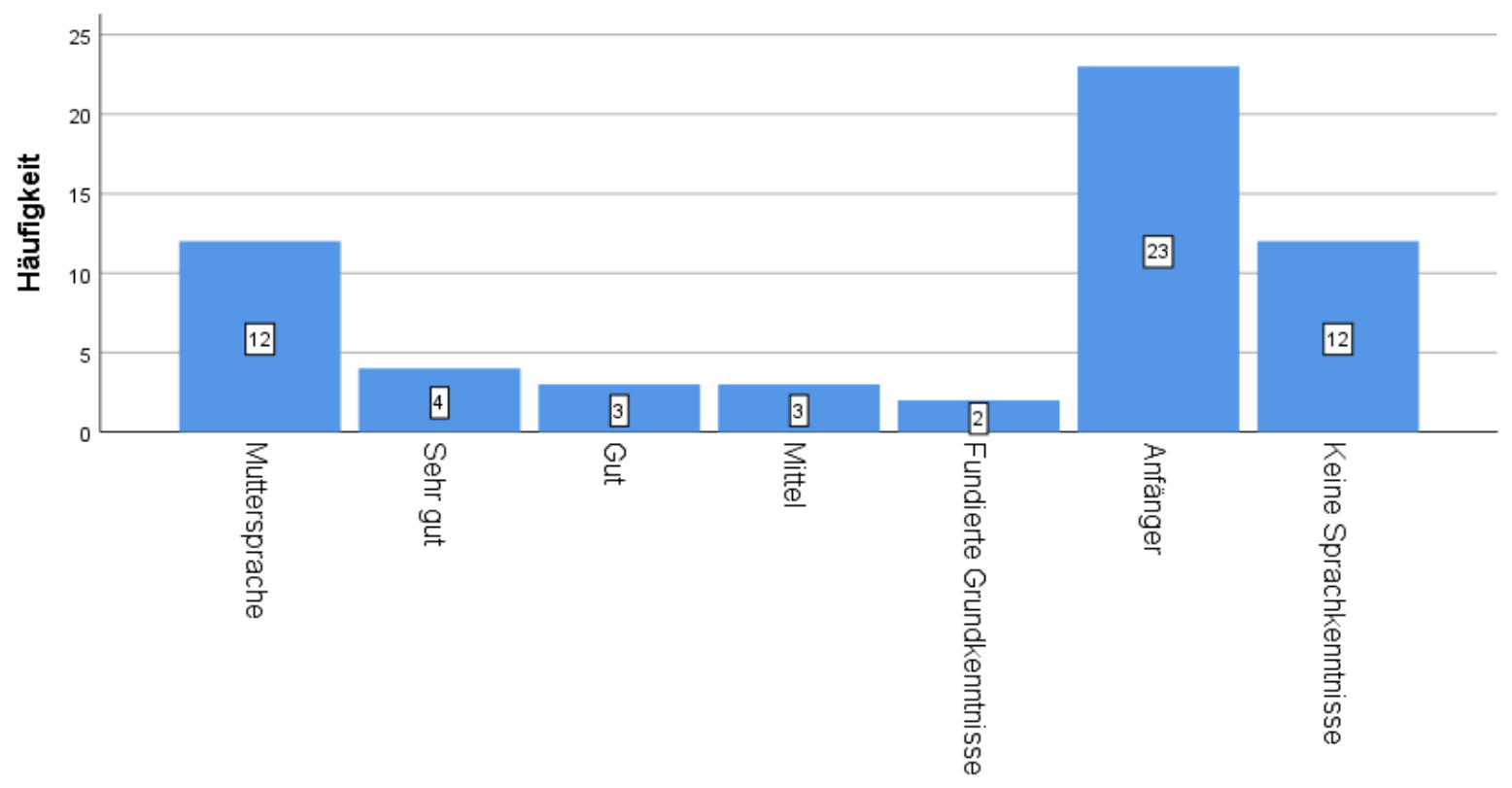

Quelle: Eigene Darstellung

Fig. 2: Nach der Werkstattwoche: „Wie schätzt du deine Polnisch-Sprachkenntnisse ein?“ Absolute Werte!

\subsubsection{Inter- und transkulturelles Verhalten}

Ferner wurden die Befragten vor der Werkstattwoche darum gebeten generelle Aussagen über

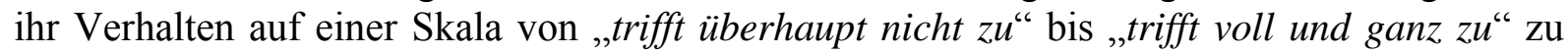
treffen, um Rückschlüsse über ihre Offenheit (eine der sechs abgefragten Dimensionen der inter- und transkulturellen Kompetenz) zu ziehen. Auf die Aussage „Ich lerne Menschen aus anderen Ländern kennen“ reagierten 16 aus der Grundgesamtheit von 62 Befragten mit „trifft voll und ganz zu“, 25 mit „,eher zutreffend“, 15 mit ,weder noch", 5 mit „,trifft eher nicht zu“ und 1 mit ,trifft überhaupt nicht zu“. (Fig. 3-Abb. 3) 


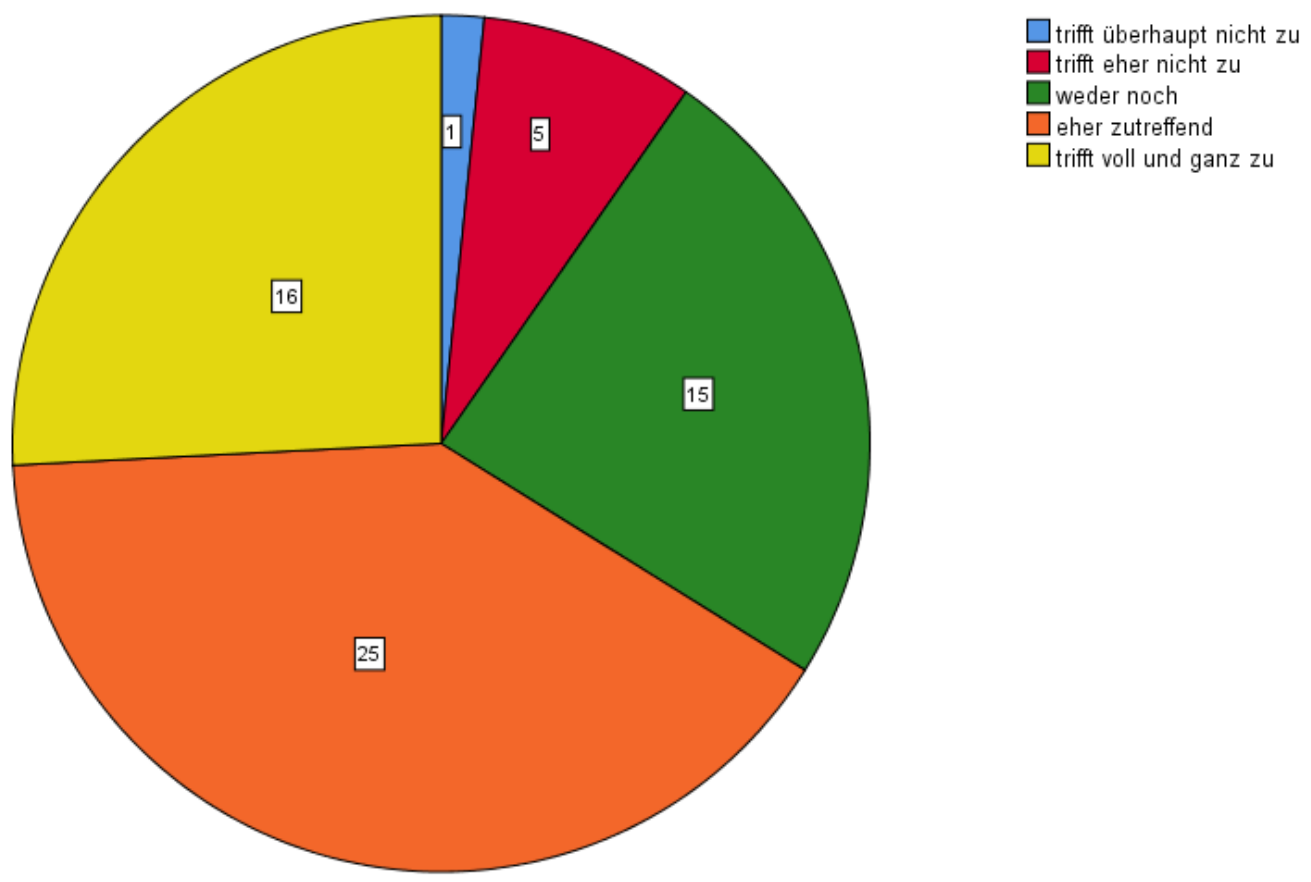

Quelle: Eigene Darstellung

Fig. 3: Vor der Werkstattwoche: „Ich lerne gerne Menschen aus anderen Ländern kennen.“ Absolute Werte!

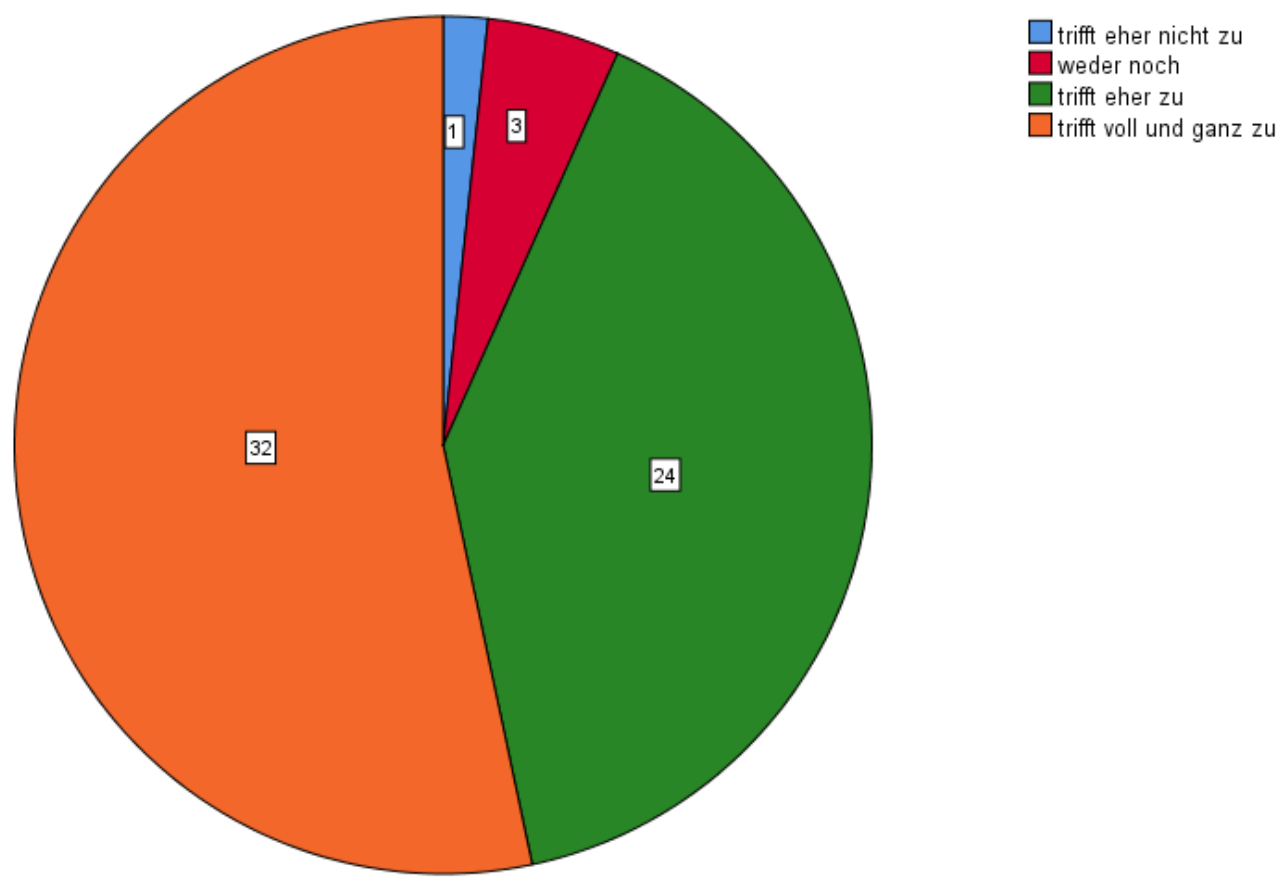

Quelle: Eigene Darstellung

Fig. 4: Nach der Werkstattwoche: „Ich lerne gerne Menschen aus anderen Ländern kennen. " Absolute Werte!

Nach der Werkstattwoche antworteten 32 aus insgesamt 60 Befragten mit ,trifft voll und ganz zu“ (um 16 Befragte mehr als vor der Woche), 24 mit „trifft eher zu“, 3 mit „,weder noch“" und 1 mit ,trifft eher nicht zu“, was den Zuwachs an Offenheit nach der Werkstattwoche belegt. (Fig. 4 - Abb. 4) 


\subsubsection{Offene Frage „Während der Werkstattwoche hat mich am meisten überrascht”}

Als letztes Beispiel der Zwischenergebnisse der quantitativen Auswertung wird die offen gestellte Frage: „Während der Werkstattwoche hat mich am meisten überrascht..." erläutert. (Fig. 5 - Abb. 5)

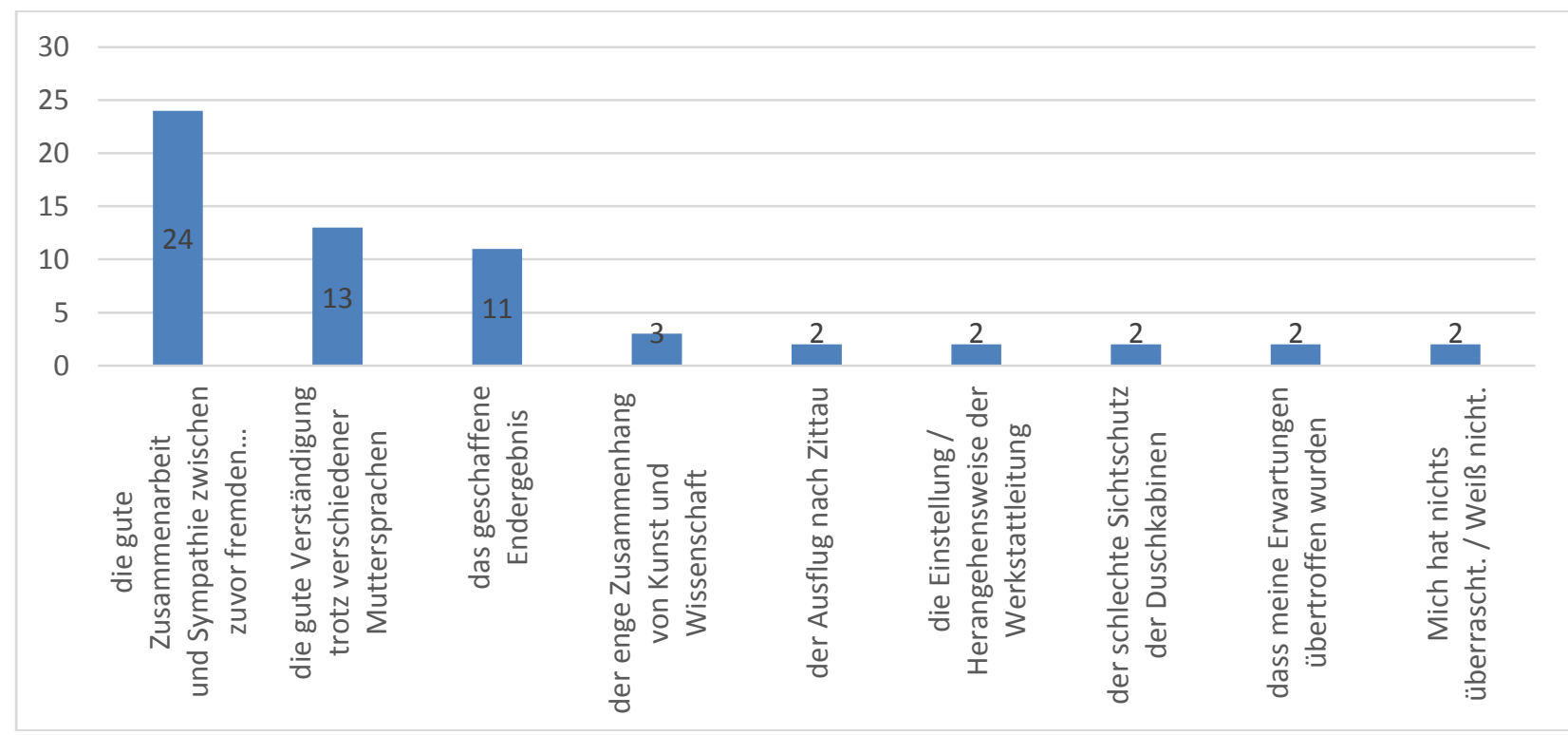

Quelle: Eigene Darstellung

Fig. 5: Nach der Werkstattwoche: „,Während der WSW hat mich am meisten überrascht ... “ Absolute Werte!

$\mathrm{Zu}$ den drei am häufigsten genannten überraschenden Sachverhalten gehören: „Die gute Zusammenarbeit und Sympathie zwischen zuvor fremden Menschen" mit 24 Nennungen, „Die gute Verständigung trotz verschiedener Muttersprachen“ mit 13 Nennungen und „Das geschaffene Endergebnis“ mit 11 Nennungen. Das Ergebnis lässt darauf schließen, dass 24 der Teilnehmer/innen der Werkstattwoche im März 2018 die Zusammenarbeit mit fremden Teilnehmer/innen (damit sind zwangsläufig die Teilnehmer/innen aus anderen Ländern gemeint, da die nationalen Gruppen sich bereits untereinander kannten) als gut eingestuft haben. Weitere 13 schätzten die sprachliche Verständigung innerhalb der internationalen Gruppen ebenfalls als gut ein.

\section{Fazit}

Aus der Analyse der vorhandenen Zwischenergebnisse ließen sich folgende Handlungsempfehlungen für die Professionalisierung des Projektes ableiten:

1. Ein Konsens über die zu erreichenden Ziele bei den Jugendlichen sowie über die Ausrichtung des Projektes ist anstrebenswert.

2. Das Potential verschiedener Erfahrungen der Werkstattleiter*innen soll über Wissenstransfer ermöglicht und gefördert werden.

Aufbauend auf den genannten Handlungsempfehlungen fanden im September und Oktober 2018 zwei Seminare für die Werkstattleiter/innen und Sprachmittler/innen statt, bei welchen der Wissenstransfer ermöglicht wurde. Bei den Seminaren wurde zudem an Lernzielbestimmung gearbeitet. Die Lernziele wurden formuliert und $\mathrm{zu}$ folgenden Überkategorien zusammengefasst [1: 37]:

- soziale Kompetenzen im interkulturellen Kontext

- Aneignung und Beschaffung von Wissen 
- Einüben des Perspektivenwechsels

- Erkennen und Überwinden von Ethnozentrismus

- Reflektieren von Situationen des interkulturellen Umgangs

- Fördern von Einstellungen und Werten

- Verbindung von Kunst und Technologie

Die veranstalteten Seminare weisen auf die Umsetzung der Handlungsempfehlungen hin, was eine gewinnbringende Zusammenarbeit zwischen einer universitären Einrichtung und informellen Bildungsträgern bestätigt. Gleichzeitig lässt sich die Kooperation als prozesshaft beschreiben, da sie aus mehreren Arbeits- und Untersuchungsschritten besteht. Des Weiteren haben die vorliegenden Zwischenergebnisse zunächst einen Orientierungscharakter für die Zweite Untersuchungsperiode.

\section{Dedikation}

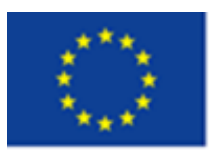

Europăische Union. Europăischer Fonds für regionale Entwicklung. Evropská unie. Evropský fond pro regionální rozvoj.

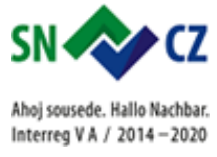

\section{Literatur}

[1] BERTELS, U.; EYLERT, S.; LÜTKES, C.; DE VRIES, S.: Ethnologie in der Schule. Eine Studie zur Vermittlung interkultureller Kompetenz. Waxman Verlag, Münster, 2004. ISBN 978-3830913382.

[2] MAYRING, P.: Qualitative Inhaltsanalyse: Grundlagen und Techniken. Beltz, Weinheim, 2003. ISBN: 978-3407257307.

[3] MOOSMÜLlER, A.: Interkulturelle Kompetenz und interkulturelle Kenntnisse. Überlegungen $\mathrm{zu}$ Ziel und Inhalt im auslandsvorbereitenden Training. In: Roth, K. (Hg.): Mit der Differenz leben. Europäische Ethnologie und interkulturelle Kommunikation. München, New York 2000, pp. 271-290.

[4] SCHMIDT, C.: „Am Material“: Auswertungstechniken für Leitfadeninterviews. In: Friebertshäuser, B.; Prengel, A. (Hrsg.): Handbuch qualitativer Forschungsmethoden in der Erziehungswissenschaft. Juventa, München und Weinheim 1997, pp. 544-567.

[5] WELSCH, W.: Transkulturalität. Zur veränderten Verfassung heutiger Kulturen. In: Schneider, I., Thomson, C. W. (Hg.): Hybridkultur: Medien, Netze, Künste. Köln und Wien, 1997, pp. 67-90.

[6] WELSCH, W.: Die veränderte Verfassung heutiger Kulturen. In: Duve, F. (Hrsg.): Sichtweisen. Die Vielheit in der Einheit. Frankfurt/Main., 1997, pp. 83-122.

[7] WELSCH, W.: Transkulturalität. Zwischen Globalisierung und Partikularisierung. In: Cesana (Hrsg.) Interkulturalität. 1999, pp. 45-72.

[8] Werkstätten. In: Lanterna Futuri. [online]. [Abrufdatum 2018-10-30] Verfügbar unter WWW https://www.lanternafuturi.net/de/werkstaetten/

[9] Interviewtranskript 1, Absatz 108.

[10] Interviewtranskript 4, Absatz 82.

[11] Interviewtranskript 5, Absatz 102.

[12] Interviewtranskript 10, Absatz 163.

Dipl.-Soz.Wiss. Sînziana Schönfelder; M.A. Emilia Socha 


\section{PROFESIONALIZACE TRINACIONÁLNÍHO SETKÁVACÍHO PROJEKTU V TROJMEZÍ D- PL-CZ NA ZÁKLADĚ VĚDECKÉ SUPERVIZE}

Projekt setkání „Umění a věda - inovativní učební metody v přsshraniční spolupráci v trojmezí D-CZ-PL“ představuje spolupráci Mezinárodního vysokoškolského ústavu (IHI) v Žitavě, Technické univerzity v Drážd’anech (D), Hillersche vily v Žitavě (D) a vědeckého centra iQLANDIA $\mathrm{v}$ Liberci $(\mathrm{CZ})$. Cílem vědeckého monitoringu je zajištění kvality prostř̌ednictvím hodnocení mezikulturních a transkulturních kompetencí zúčastněné mládeže, jakož i kvalitativního průzkumu s ohledem na požadavky dalšího vzdělávání zúčastněných. Tento článek popisuje první předběžné výsledky kvalitativních a kvantitativních průzkumů prováděných vědeckým monitoringem a také následné doporučení $\mathrm{k}$ opatření.

\section{Professionalization of the Encounter Project in the Tri-Point OF D-PL- CZ ON THE BASIS OF SCIENTIFIC SUPERVISION}

The encounter project "Art and Science - Innovative methods of learning in the cross-border collaboration in the tripoint of D-CZ-PL" represents cooperation of International Institute (IHI) Zittau - Technical University of Dresden (D), the Hillersche Villa e. V. in Zittau (D) and the science center iQLANDIA in Liberec (CZ). The objective of the scientific monitoring is the quality assurance by means of the evaluation of inter- and transcultural competence of the participating youth as well as a qualitative survey with respect to further training requirements of the participating multipliers. This article describes the first preliminary results of the qualitative and quantitative surveys conducted by the scientific monitoring as well as the resulting recommendation for action.

\section{PROFESJONALIZACJA TRÓJSTRONNEGO PROJEKTU DOTYCZĄCEGO SPOTKAŃ NA DE- PL-CZ TRÓJSTYKU W OPARCIU O MONITORING NAUKOWY}

Projekt spotkań pn. „Sztuka i nauka - innowacyjne metody nauczania we współpracy transgranicznej na trójstyku DE-CZ-PL" obejmuje współpracę Międzynarodowego Instytutu Uniwersyteckiego (IHI) w Zittau, Uniwersytetu Technicznego w Dreźnie (DE), Hillershe Villa w Zittau (DE) i Centrum Nauki iQLANDIA w Libercu (CZ). Celem monitoringu naukowego jest zapewnienie jakości w drodze oceny kompetencji międzykulturowych i transkulturowych uczestniczącej młodzieży a także badania jakościowe prowadzone pod kątem wymogów co do dalszego kształcenia uczestników. W niniejszym artykule opisano pierwsze wstępne wyniki badań jakościowych $\mathrm{i}$ ilościowych prowadzonych $\mathrm{w}$ formie monitoringu naukowego oraz wynikające z nich zalecane działania. 